\title{
A HIDDEN CLASS OF BE STARS?
}

\author{
P.A. ZAAL \\ Kapteyn Astronomical Institute, Groningen \\ L.B.F.M. WATERS \\ Astronomical Institute 'Anton Pannekoek', Amsterdam \\ SRON Laboratory for Space Research, Groningen \\ J.M. MARLBOROUGH \\ Astronomy Department, University of Western Ontario, London, Canada \\ and \\ T.R. GEBALLE \\ Joint Astronomy Centre, Hilo, Hawaii, USA
}

\section{Introduction}

Recently, $\operatorname{Br} \alpha$ and $\operatorname{Br} \gamma$ emission was detected in the infrared spectrum of the B0.2V star $\tau$ Scorpii, without noticeable emission in $\mathrm{H} \alpha$ (Waters et al. A\&A 272, L9-L12, 1993). Here we present simple HI recombination line calculations in the infrared and in the optical that demonstrate that there could be a class of B stars with low-density discs, a factor of 100 lower in density compared to normal Be stars, which may have escaped detection so far.

\section{The disc model}

We carried out a parameter study for $4 \mathrm{~B}$ stars of different $\mathrm{T}_{\text {eff }}$ to find out under which conditions a hot star with a circumstellar disc can have IR emission lines without obvious $\mathrm{H} \alpha$ emission. For the underlying photospheric continuum (from Kurucz, 1979) we used $\mathrm{T}_{\text {eff }}=30000,23000,15000$ and $12000 \mathrm{~K}$ and $\mathrm{R}_{\star}=$ $6,4.3,3$ and $2.5 \mathrm{R}_{\odot}$ for resp. spectral type B0, B2, B5 and B8. We used a simple disc model which consists of a disc with a opening angle $\theta$, with $\theta=5^{\circ}$, and a density distribution, $\rho(r)=\rho_{0}\left(r / R_{\star}\right)^{-2.5}$ inside the disc. For mass continuity this corresponds to a velocity , $v(r)=v_{0} \sqrt{r / R_{\star}}$. We adopted a Keplerian rotation, $v_{\phi}(r)=v_{\phi, 0} \sqrt{R_{\star} / r}$, for the disc where $v_{\phi, 0}=0.7 \cdot v_{b r}$, with a breakup velocity, $v_{b r}$ of about $800 \mathrm{~km} / \mathrm{s}$ for a B0V star.

The $\mathrm{H} \alpha$ line was calculated solving the equations of stastistical equilibrium for the levels 1 to 4 for a gas consisting of pure H (Marlborough J.M., ApJ 156, 135, 1969), taking into account the underlying photospheric absorption line. We further assume that the disc is isothermal at a temperature of $0.6 \cdot \mathrm{T}_{\text {eff }}$. The IR recom- 
bination lines were calculated with an optically thin approximation. It calculates the line intensity of a volume of gas with density $\rho(r)$ and temperature T:

$$
I_{\text {approx }}=E M \cdot \alpha_{\text {line }, e f f} \cdot h \nu_{\text {line }}
$$

With EM the emission measure of the emitting gas, $h \nu_{\text {line }}$ the energy of the line and $\alpha_{\text {line,eff }}$ the effective recombination coefficient for case B from Hummer and Storey (MNRAS, 224, 801, 1987).

The approximate line intensity can be compared to numerical calculations. The difference between the two is mostly due to optical depth effects. The boundaries in density for our possible new class of Be stars are due to two facts. The upper limit is due to the emission feature which appears in $\mathrm{H} \alpha$. One gets the already known Be stars. The lower limit is due to the fact that no line emission in the infrared is visible any more below that density. To enlarge the density range for detection of the disc in the IR we have to find the HI line in the infrared with the strongest line over continuum ratio. Between $2 \mu \mathrm{m}$ and $50 \mu \mathrm{m}$ we find a maximum for the 10-09 $\mathrm{HI}$ line transition at $38.9 \mu \mathrm{m}$.

The line over continuum ratio of the IR line depends strongly on the inclination angle of the disc. A disc viewed edge on will give fewer detectable emission lines. The dashed lower limit and solid lower in figure 1 shows the difference between resp. pole on and edge on.

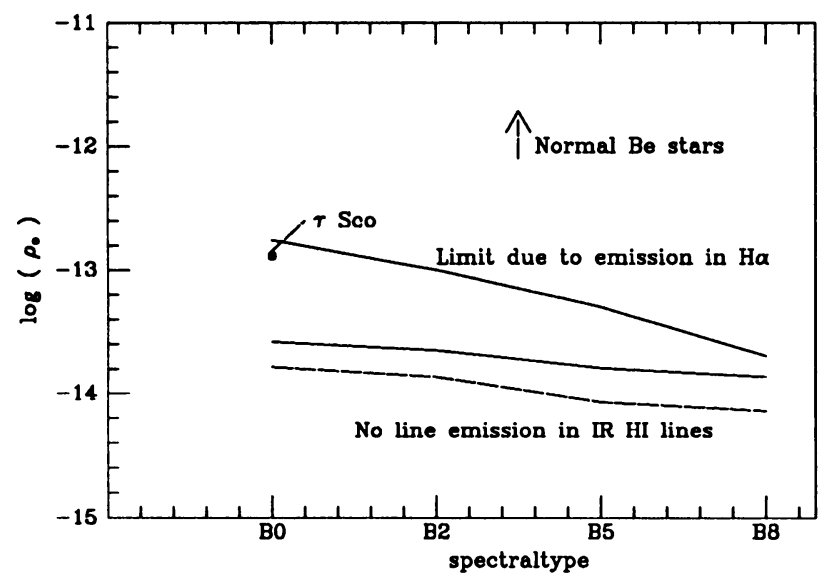

Fig. 1. The density range for low-density discs.

\section{Conclusions}

We propose that $\tau$ Sco is a member of a new class of B stars with low-density circumstellar gas, possibly a disc. The HI (10-09) line at $38.9 \mu \mathrm{m}$ is most sensitive to low density circumstellar gas. These stars will be great targets for ISO. 